\title{
Low-Cost Hybrid Analog-Digital Beamformer Evaluation in Spectrum Sharing Systems
}

\author{
Miguel Ángel Vázquez ${ }^{1}$, Xavier Artiga ${ }^{1}$, Ana I. Pérez-Neira ${ }^{1,2}$ \\ ${ }^{1}$ Centre Tecnològic de les Telecommunicacions de Catalunya: CTTC, Castelldefels, Spain, xartiga@cttc.cat \\ ${ }^{2}$ Universitat Politècnica de Catalunya: UPC, Dept. of Signal Theory and Communications, Barcelona, Spain
}

\begin{abstract}
This paper evaluates different analog-digital beamforming solutions for future spectrum sharing mm-wave scenarios. In contrast to sub-6 $\mathrm{GHz}$ multiantenna schemes where all-digital solutions provide an excellent performance-cost tradeoff, in the mm-wave bands where a very large number of antennas is required, all-digital designs cannot be deployed due to their cost and complexity. In order to solve this problem, subarray solutions are conceived such that a reduced number of radiofrequency chains are simultaneously connected to different antennas through an analog beamforming network formed by phase shifters (i.e. with no amplitude control). Different connectivity solutions are evaluated; namely, full-connected, localized and interleaved considering that either the phase shifters have full resolution or only one control bit. As reported in the paper, while for the full resolution case the same performance is obtained for all connectivity schemes, in case the phase shifters have one control bit, differences show up. The numerical evaluation is done with an alternating feasible point pursuit successive convex approximation (FPP-SCA) optimization which yields to efficient solutions even for this non-convex optimization problem.
\end{abstract}

Index Terms-Hybrid analog-digital beamforming, mm-wave beamforming, interference rejection.

\section{INTRODUCTION}

Millimeter wave (mm-wave) communications are foreseen as one of the main technology enablers for future $5 \mathrm{G}$ networks since they can cope with the required higher throughputs thanks to their large relative bandwidths. However, due to the large path-loss experienced at those frequencies, mm-wave communications must rely on high gain steerable antennas in both access and backhaul networks. In the backhaul case, large beamforming arrays are being investigated jointly with interference mitigation techniques for certain spectrum sharing mm-wave scenarios [1], [2]. As a matter of fact, digital beamforming (DBF) antenna arrays offer the best performance in terms of beamforming capabilities. Unfortunately, the high gain requirements of mm-waves translate in high number of antennas that make the DBF solution unaffordable in terms of cost and complexity. At the other end, analog beamforming $(\mathrm{ABF})$ has a limited processing capability (i.e. the one offered by the analog components) and their implementations become bulky and lossy when the number of antennas is large. In this context, current investigations have been focusing on hybrid analog-digital beamforming (HADBF) which allows balancing the number of RF chains, the size of the analog beamforming networks and the processing capabilities.

Most relevant works on this area are surveyed in [3]. Interestingly, in many cases a full-array configuration for the analog beamformer has been considered in spite of their extreme implementation complexity due to the connection of each RF chain with each antenna element. Moreover, the insertion loss of the power combiners at the input of each antenna element is usually neglected what results in an overestimation of the full-array performance [4].

In this paper, we evaluate the performance of different HADBF solutions, including the full-array configuration with a proper insertion loss modelling as well as localized and interleaved subarrays. Moreover, as a lower cost solution, we consider the use of aggressive quantization down to 1 bit for the analog phase shifters. In contrast with most of the works reviewed in [3], we address the cognitive beamforming optimization [5]. Therefore, our aim is to maximize the gain at a desired angle while limiting the gain at interfered angles below a given threshold.

HADBF optimization for spectrum sharing system have been investigated recently by the authors [6], [7]. To the best of authors knowledge the works in [6], [7] are the first ones devoted to the HADBF cognitive optimization. Previously, [5] presented the optimization problem when an all-digital transceiver is considered. The proposed method is based on feasible point pursuit successive convex approximation (FPPSCA) [8], a method for approximately solve non-convex quadratically constraint quadratic programs (QCQP) without any a prior knowledge of a feasible point. Precisely, we propose an alternating optimization that iteratively solves nonconvex QCQPs with FPP-SCA. This method is not depicted in this paper but the reader can refer to [9] for further details.

The paper is organized as follows: Section II introduces the mathematical system model; Section III details the HADBF solutions considered; Section IV explains the optimization method used; and Section V shows the numerical simulation results. Finally, Section VI concludes the paper.

\section{System Model}

Let us assume a spectrum sharing backhauling network where a transmitter is equipped with $Q$ antennas. The receive signal of an intended user can be modelled as

$$
y=\gamma_{d} \sqrt{P} \mathbf{h}_{d}^{H} \mathbf{v} s_{d}+n_{d},
$$

where $\mathbf{v} \in \mathbb{C}^{Q \times 1}$ is the transmit beamforming vector, $P$ is the transmit power, $\mathbf{h}_{d} \in \mathbb{C}^{Q \times 1}$ is the vector channel, $\gamma_{d}$ is the path-loss between the transmitter and the intended receiver 
and $n_{d}$ is the additive white zero mean unit variance white Gaussian noise. The transmitter sends a unit norm symbol and it is denoted by $s_{d}$. Similarly to the intended user, we assume there are a set of $K$ interfered users. The channel vector of the $k$-th interfered user is denoted by

$$
\mathbf{h}_{i_{k}}, \quad k=1, \ldots, K
$$

For the channel matrix we assume the model provided in [10] which addresses multiantenna microwave backhaul links deployed in 'above roof top' scenarios. In particular, the channel matrix can be written as

$$
\mathbf{h}=\sum_{n=1}^{S+1} \alpha_{n} \mathbf{a}_{k}\left(\theta_{n}, \phi_{n}\right),
$$

where $S$ is the total number of scatters, $\alpha_{n}$ the complex gain of the $n$-th scatter, $\mathbf{a}_{k} \in \mathbb{C}^{Q \times 1}$ is the steering vector of the transmit antenna array. The steering vector depends on the angles of departure (AoD), $\theta_{n}, \phi_{n}$. For $n=1$ it is consider that the channel is deterministic and it can be obtained via a geometrical reasoning. Precisely, it is assumed that $\alpha_{1}=1$ and $\theta_{1}, \phi_{1}$, can be computed by knowing the relative position of the transmitter and the receiver. For $n>1$, the channel offers a random behaviour based on the first ray $(n=1)$. In this case, the amplitude values can be modelled as

$$
\alpha_{n}=A_{n} e^{\psi_{n} j}
$$

where $A_{n}$ is Rayleigh distributed with mean $\gamma_{l} / 10$ and $\psi_{n}$ is uniformly distributed from 0 to $2 \pi$. Moreover, it is assumed that the number of scatters is fixed and it is $S=4$. Finally, the AoDs for the different scatters $n>1$ are perturbed by an additive Gaussian random variable of zero mean and 5 degrees of standard deviation. The steering vector a depends on the antenna array structure and the element spacing. In this paper we consider the simplest array structure, i.e. the uniform linear array (ULA).

\section{Hybrid AnAlog-Digital Structures}

Figures 1, 2 and 3 depict the three HADBF schemes evaluated in this paper. Targeting low cost solutions, it is assumed that, in all cases, the analog beamformers only perform phase control through the use of phase shifters. In a first step full resolution phase shifters are considered. Then the impact of aggressive phase quantization is studied assuming low cost 1-bit phase shifters. The localized subarray (Fig. 1) is the traditional corporate feed network in which each RF feeds a contiguous set of antennas. In this way each feed only uses part of the total aperture, so its array factor suffers from beam widening and reduced gain. In the interleaved scheme (Fig. 2 ), each RF feeds a non-contiguous subarray. In this case, each feed uses the whole aperture but with large spacing between elements, which creates grating lobes in its array factor. Finally, in the full array configuration (Fig. 3) each RF feeds all array elements. To this end, the signal coming from each RF is divided in $Q$ branches and goes through a phase shifter. Then a power combiner at the input of each antenna

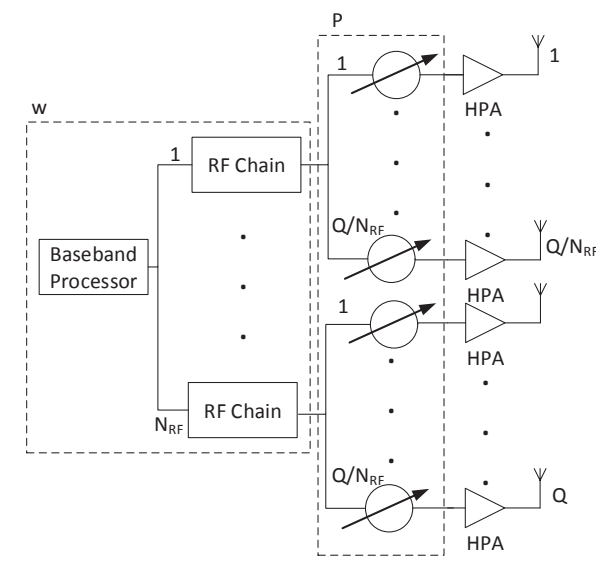

Fig. 1. Localized hybrid analog-digital solution.

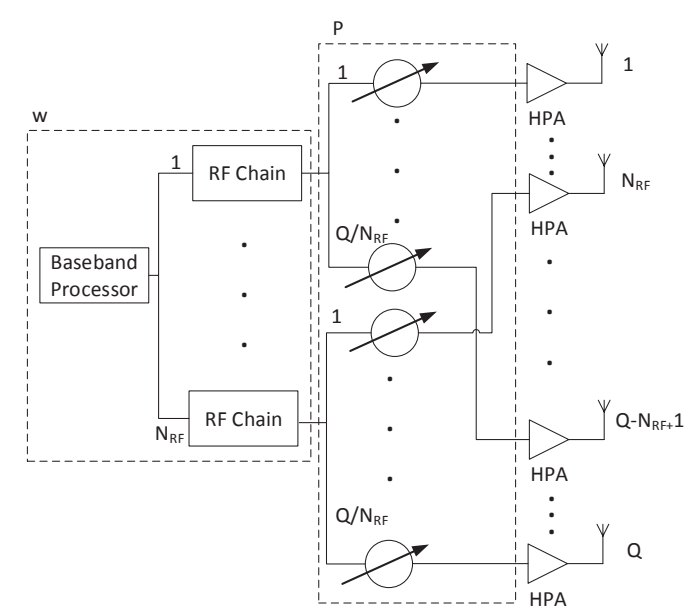

Fig. 2. Interleaved hybrid analog-digital solution.

combines the $N_{R F}$ phase shifted signals intended for each antenna. This solution requires $Q N_{R F}$ phase shifters, $N_{R F}$ $Q$-way power dividers, $Q N_{R F}$-way power combiners and the interconnection of all these modules. As a result its cost and complexity may become unaffordable as the number of antennas goes large, as required in mm-wave communications. On the contrary, subarray schemes only need $Q$ phase shifters and $N_{R F}\left(Q / N_{R F}\right)$-way power dividers. In this case, the RF crossings required in the interleaved subarray may increase the size and complexity of the beamforming network with respect to the localized one, which must be seen as the most cost effective solution. Moreover, the full array beamforming network may suffer larger insertion loss than the subarrays. This is due to the presence of the power combiners which ideally present an insertion loss of $10 \log \left(N_{R F}\right)$. This loss is only totally compensated in the case that the signals to be combined are equal. In order to properly model this, we consider that 


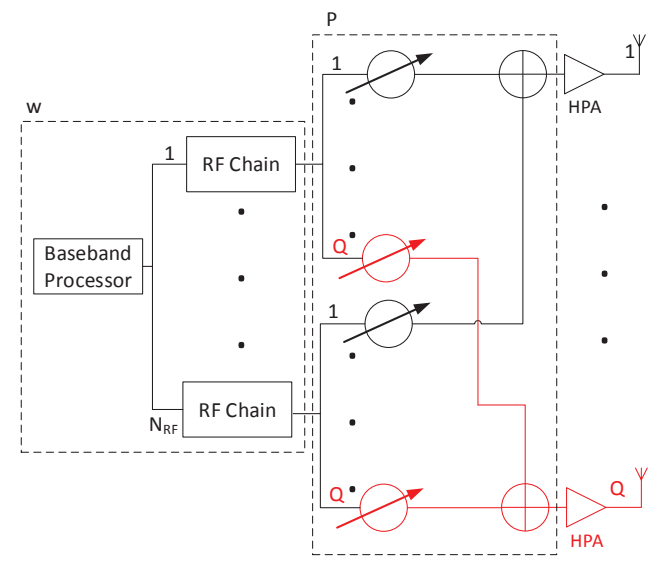

Fig. 3. Full connected hybrid analog-digital solution.

the $Q N_{R F}$ paths from each RF to each power combiner are affected by a $\frac{1}{\sqrt{Q N R F}}$ amplitude factor in the full array scheme, whereas the Q paths of the subarray schemes experience only a factor of $\sqrt{\frac{N_{R F}}{Q}}$. Note that here only the ideal insertion loss of dividers and combiners are taken in account. Other sources of loss such as phase shifters or Ohmic losses would only scale the results and are neglected for simplicity. As detailed in the next section, for a fair comparison we apply power constraints at the input of the analog beamforming networks, thus the power amplifiers shown in Fig.1-3 are assumed to provide a constant gain which is neglected in the power modelling.

An a priori evaluation of the three schemes for the single stream scenario considered in this paper yields the following conclusions:

- With full resolution phase shifters the analog beamformers of the three solutions can provide full phase control. In contrast, only the full array analog beamfomer provides full amplitude control. This occurs when the powers at the output of the digital beamformers are equal, so the amplitude is controlled by the combination of two equal amplitude phasors. The subarray schemes can only control the amplitude in subarray blocks by changing the powers at the outputs of each RF chain.

- With 1-bit phase shifters, each antenna in the subarray schemes can present two phase states: $\theta$ or $\theta+\pi$, being $\theta$ the phase at the output of the RF chain to which the antenna is connected. In contrast, each antenna in the full array configuration can present up to $2^{N_{R F}}$ phase states (if the phases at the RF chains outputs are all different).

\section{Optimization PRoblem}

This paper focuses on the interference-constraint optimization problem where the array gain is maximized in the intended user channel vector while kept under a certain threshold to the non-intended one. For the full analog or full digital case, the optimization problem becomes

$$
\begin{aligned}
& \underset{\mathbf{v}}{\operatorname{maximize}}\left|\mathbf{h}_{d}^{H} \mathbf{v}\right|^{2} \\
& \text { subject to } \\
& \left|\mathbf{h}_{i_{k}} \mathbf{v}\right|^{2} \leq \epsilon_{k} \quad k=1, \ldots, K, \\
& \mathbf{v} \in \mathcal{A},
\end{aligned}
$$

where $\epsilon_{k}$ is the maximum acceptable interference level for the $k$-the interference. The feasible set $\mathcal{A}$ varies whenever we target the full-digital optimization

$$
\mathcal{A}_{\text {digital }}:\left|[\mathbf{v}]_{i}\right|^{2} \leq P_{\max } \quad i=1, \ldots, Q,
$$

or a full-analog design

$$
\mathcal{A}_{\text {analog }}:\left|[\mathbf{v}]_{i}\right|^{2}=P_{\max } \quad i=1, \ldots, Q,
$$

where $P_{\max }$ is the maximum available power for each antenna. For the analog case and due to the equality constraints, the optimization problem in (5) is non-convex and it requires advanced techniques for approximately solve it [6], [8]. Concretely, we propose a variation of FPP-SCA in [8]. For more details, the reader can refer to [11]. When an hybrid analogdigital beamforming design is targeted, the optimization problem shall be reconsidered. Mathematically, it can be modelled as follows

$$
\begin{aligned}
& \underset{\mathbf{P}, \mathbf{w}}{\operatorname{maximize}}\left|\mathbf{h}_{d}^{H} \mathbf{P} \mathbf{w}\right|^{2} \\
& \text { subject to } \\
& \left|\mathbf{h}_{i_{k}} \mathbf{P} \mathbf{w}\right|^{2} \leq \epsilon_{k} \quad k=1, \ldots, K, \\
& \mathbf{P} \in \mathcal{P}, \\
& \mathbf{w} \in \mathcal{W},
\end{aligned}
$$

where $\mathbf{P} \in \mathbb{C}^{Q \times N_{R F}}$ refers to the analog processing part and $\mathbf{w} \in \mathbb{C}^{N_{R F} \times 1}$ to the digital processing part. Depending on the considered cases, we have different alternatives to $\mathcal{P}$ and $\mathcal{W}$. In particular, two different power constraint alternatives are considered. A per-RF power constraint that limits the maximum power injected by each RF chain to the analog beamformer, and a sum-power constraint that limits the total power injected to it.

$$
\begin{gathered}
\mathcal{W}_{\text {per-RF }}:\left|[\mathbf{w}]_{i}\right|^{2} \leq P_{\text {max-RF }} \quad i=1, \ldots, N_{R F}, \\
\mathcal{W}_{\text {sum-RF }}:\|\mathbf{w}\|^{2} \leq P_{\max -\mathrm{RF}} N_{R F},
\end{gathered}
$$

where $P_{\text {max-RF }}$ is the maximum available power per RF chain. It is important to remark the substantial difference between this power control and other approaches such as [12], [13], [14]. While in this paper we fix our attention to the maximum power delivered by to the analog beamformer, in [12], [13], [14] a flexible sum-power interchange between antennas is considered with no restriction over the maximum delivered power to the analog beamformer. In this context, this paper proposal is able to fairly evaluate the beamforming network efficiency as it keeps the delivered power to the analog beamformer under a certain common threshold for all considered architectures. 
For the analog part, we consider the following feasible sets such as

$$
\begin{gathered}
\mathcal{P}_{\text {full }}:\left|[\mathbf{P}]_{m, n}\right|^{2}=\alpha, \\
\mathcal{P}_{\text {interleaved }}:\left|[\mathbf{P}]_{m, n}\right|^{2}=\left[\mathbf{1}_{\lambda} \otimes \beta \mathbf{I}_{N_{R F}}\right]_{m, n}, \\
\mathcal{P}_{\text {localized }}:|[\mathbf{P}]|^{2}=\left[\beta \mathbf{I}_{N_{R F}} \otimes \mathbf{1}_{\lambda}\right]_{m, n},
\end{gathered}
$$

for $m=1, \ldots, Q \quad n=1, \ldots, N_{\mathrm{RF}}$ and

$$
\lambda=\frac{Q}{N_{R F}},
$$

which is assumed to be an integer value. Moreover,

$$
\alpha=\frac{1}{Q N_{R F}}, \quad \beta=\frac{N_{R F}}{Q},
$$

which are the array losses as described in the previous section. The optimization problem in (8) is a difficult non-convex QCQP problem. The authors propose a method for solving it via an alternating optimization and FPP-SCA. The method is described in [9] and it presents similar performance to [7] while substantially reducing the computational time.

\section{NumericAl RESUlts}

This section describes the performance evaluation of the different HADBF schemes presented in this paper. In all cases, only one interference has been considered and the we set $\epsilon=-30 \mathrm{~dB}$. In all evaluations we depict the empirical cumulative distribution function (ECDF) based on 200 Monte Carlo runs. In each run, the line-of-sight angle of the intended and the interference are randomly uniformly placed between -90 and 90 degrees and the scatters are formed by the mentioned backhaul channel model as described in Section II. Moreover, the figure of merit presented is the array gain

$$
\text { Array Gain }[\mathrm{dB}]=20 \log \left(\left|\mathbf{h}_{d}^{H} \mathbf{P} \mathbf{w}\right|\right) \text {. }
$$

Note that both array gain and interference level take in consideration the contributions of all four scatterers. For the sake of simplicity an uniform linear array structure has been considered. First, Figure 4 shows the array gain with both an alldigital beamforming with per antenna power constraints and an all-analog phase-only design, both with $Q=8$ antennas. It can be observed from the figure that both schemes show a very similar performance. In other words, the pure phase control (i.e. analog beamforming) offers almost the same performance of phase and amplitude control (i.e. digital beamforming solution). Therefore, in case the analog beamforming network is formed by full resolution phase shifters all HADBF schemes will show almost the same performance behavior since all provide full phase control as discussed in Section III. In this context, the designer shall opt to the localized scheme as it is the one with lowest complexity and cost. On the other hand, in the extreme low cost case in which the analog beamforming network can only set 1 or -1 values to their phase shifters (i.e. one bit control), we observe a substantial difference between the all-digital case and the all-analog one bit solution. This is depicted in Figure 5 where the HADBF solutions are also

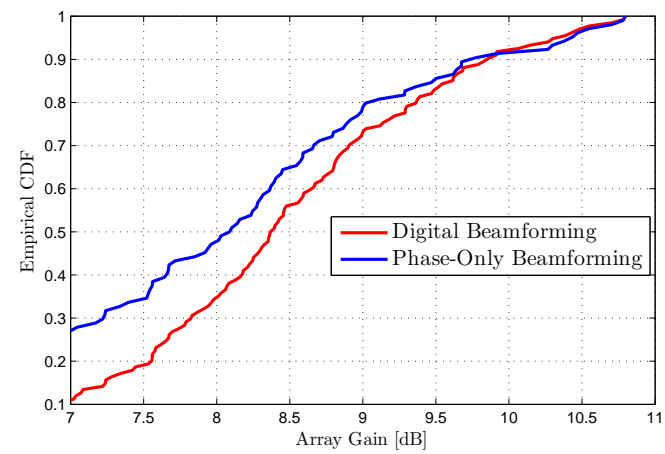

Fig. 4. Digital versus analog phase-only beamforming with $Q=8$ antennas and one interference.

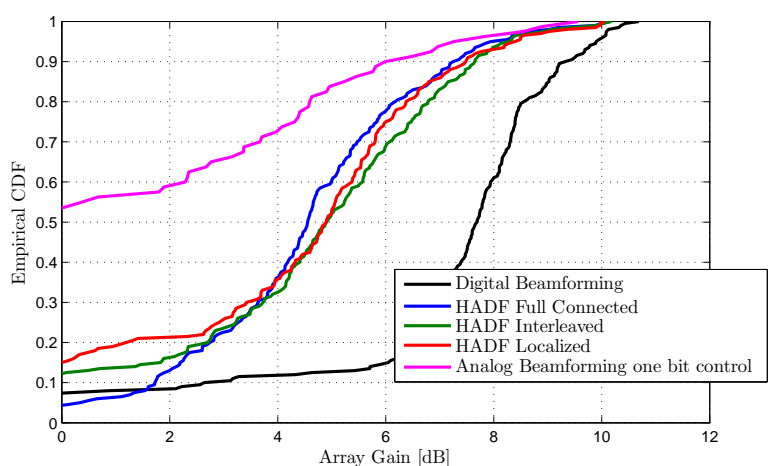

Fig. 5. Array gain empirical CDF comparison with per-RF power control with $Q=8$ and $N_{R F}=2$ with one interference randomly located.

plotted considering the per-RF power control and $Q=8$, $N_{R F}=2$. Remarkably, all HADBF solutions with one bit phase shifters only present a $2.5 \mathrm{~dB}$ performance loss with respect to the full resolution beamforming solution in the $50 \%$ percentile. No relevant differences are observed among them. The full connected solution performs slightly worse in high array gain regions and rather better in low gain regions. Note that this last region correspond to intended users close to \pm 90 degrees, which are affected by beam widening, or to very close intended and interfered locations. For the sum-RF solution, Figure 6 presents the results and the same conclusion can be drawn as for the per-RF digital power control. In light of the results, again the designer shall opt to implement the scheme with lowest complexity: the localized sub-array solution.

We now evaluate a larger antenna array with $Q=32$ and $N_{R F}=8$. For this, we place the interference value closer to the intended direction: uniformly distributed among -15 and 15 degrees close to the intended line-of-sight direction. The results are reported in Figure 7. Impressively, the array gain offered by the hybrid solutions is very close to the alldigital beamforming even though we only use one bit control phase shifters. Moreover, the performance gain compared to the analog beamforming design is very large. Among the different HADBF schemes, the full connected solution offers a larger array gain over all possible array gain values. A 


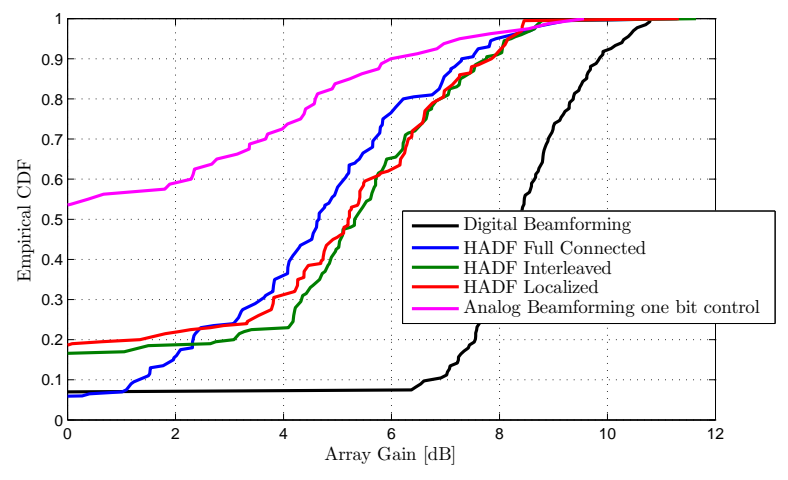

Fig. 6. Array gain empirical CDF comparison with sum-RF power control with $Q=8$ and $N_{R F}=2$ with one interference randomly located.

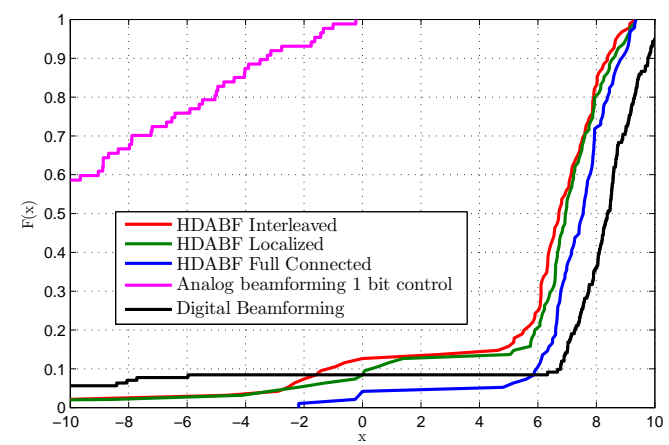

Fig. 7. Array gain empirical CDF comparison with sum-RF power control with $Q=32$ and $N_{R F}=8$ with one interference placed close to the intended user AoD.

possible explanation for this change of behavior, is that with the antenna increase, the number of possible phase states of the full connected solution also increases, as discussed in Section III, what permits balancing the also larger insertion losses. However, the performance gain of the full connected scheme over the subarray solution is so low that cannot justify its implementation cost and complexity. Again, the localized subarray solution offers the best performance cost trade-off.

\section{CONCLUSION}

This paper evaluates different HADBF network solutions from the cost and performance perspective considering a tentative spectrum sharing application. All schemes are evaluated via an alternating FPP-SCA method which is able to yield to efficient solutions yet preserving a low computational complexity. The results show that when full resolution phase shifters are considered, the performance of all-analog and alldigital schemes are very similar, thus HADBF only provides a balance in terms of complexity but not in performance. In contrast, when low cost 1-bit phase shifters are used, HDABF permits achieving a performance close to all-digital beamforming and significantly better than all-analog schemes. Among the different HADBF solutions, very similar performances are observed. Therefore, for the considered scenario, it is concluded that the localized subarray scheme provides the best cost effective solution. Note that the presented results are based on a given optimization method that does not yield to a global optimal solution. Such solution is still an open problem.

\section{ACKNOWLEDGMENT}

This work has received funding from the European Unions Horizon2020 research and innovation programme under grant agreement No 645047(SANSA); the Spanish Ministry of Economy and Competitiveness (Ministerio de Economia y Competitividad) under project TEC2014-59255-C3-1-R (ELISA); and from the Catalan Government (2014SGR1567 and 2014SGR1551)

\section{REFERENCES}

[1] T. S. Rappaport, S. Sun, R. Mayzus, H. Zhao, Y. Azar, K. Wang, G. N. Wong, J. K. Schulz, M. Samimi, and F. Gutierrez, "Millimeter Wave Mobile Communications for 5G Cellular: It Will Work!" IEEE Access, vol. 1, pp. 335-349, 2013.

[2] X. Artiga, J. N. nez Martínez, A. Pérez-Neira, G. Lendrino, J. Faré and G. Ziaragkas, "Terrestrial-Satellite Integration in Dynamic 5G Backhaul Networks," in 2016 8th Advanced Satellite Multimedia Systems Conference and the 14th Signal Processing for Space Communications Workshop (ASMS/SPSC), Sept 2016, pp. 312-317.

[3] S. Kutty and D. Sen, "Beamforming for Millimeter Wave Communications: An Inclusive Survey," IEEE Communications Surveys Tutorials, vol. 18, no. 2, pp. 949-973, Secondquarter 2016.

[4] A. J. G. Rodriguez, V. Venkateswaran, P. Rulikowski, and C. Masouros, "Hybrid Analog-Digital Precoding Revisited under Realistic RF Modeling," IEEE Wireless Communications Letters, vol. PP, no. 99, pp. 1-1, 2016.

[5] R. Zhang and Y. C. Liang, "Exploiting Multi-Antennas for Opportunistic Spectrum Sharing in Cognitive Radio Networks," IEEE Journal of Selected Topics in Signal Processing, vol. 2, no. 1, pp. 88-102, Feb 2008.

[6] M. A. Vázquez, L. Blanco, A. Pérez-Neira, and M. A. Lagunas, "Phaseonly transmit beamforming for spectrum sharing microwave systems," in Smart Antennas (WSA), 2016 International ITG Workshop on, March 2016, pp. 1-8.

[7] M. A. Vázquez, L. Blanco, X. Artiga, and A. Pérez-Neira, "Hybrid analog-digital transmit beamforming for spectrum sharing satelliteterrestrial systems," in 2016 IEEE 17th International Workshop on Signal Processing Advances in Wireless Communications (SPAWC), July 2016, pp. 1-5

[8] O. Mehanna, K. Huang, B. Gopalakrishnan, A. Konar, and N. D. Sidiropoulos, "Feasible Point Pursuit and Successive Approximation of Non-Convex QCQPs,” IEEE Signal Processing Letters, vol. 22, no. 7, pp. 804-808, July 2015.

[9] M. A. Vázquez, L. Blanco, and A. I. Pérez-Neira, "Hybrid AnalogDigital Transmit Beamforming for Spectrum Sharing Backhaul Systems," in preparation, 2016.

[10] A. Maltsev and et al, "D5.1 - Channel Modeling and Characterization," MiWEBA Project (FP7-ICT-608637), Public Deliverable, Jan. 2014.

[11] M. A. Vázquez, L. Blanco, and A. I. Pérez-Neira, "Spectrum Sharing in Hybrid Satellite-Terrestrial Backhaul Systems via Phase-Only Beamforming," in preparation, 2016.

[12] H. Ghauch, T. Kim, M. Bengtsson, and M. Skoglund, "Subspace Estimation and Decomposition for Large Millimeter-Wave MIMO Systems," IEEE Journal of Selected Topics in Signal Processing, vol. 10, no. 3 , pp. 528-542, April 2016.

[13] O. E. Ayach, S. Rajagopal, S. Abu-Surra, Z. Pi, and R. W. Heath, "Spatially Sparse Precoding in Millimeter Wave MIMO Systems," IEEE Transactions on Wireless Communications, vol. 13, no. 3, pp. 14991513, March 2014

[14] F. Sohrabi and W. Yu, "Hybrid Digital and Analog Beamforming Design for Large-Scale Antenna Arrays," IEEE Journal of Selected Topics in Signal Processing, vol. 10, no. 3, pp. 501-513, April 2016. 